\title{
Classification of extended Clifford algebras
}

\author{
N. G. Marchuk
}

September 24, 2018

nmarchuk@mi.ras.ru

Steklov Mathematical Institute of RAS, Gubkina st.8, Moscow, 119991, Russia

\begin{abstract}
Considering tensor products of special commutative algebras and general real Clifford algebras, we arrive at extended Clifford algebras. We have found that there are five types of extended Clifford algebras. The class of extended Clifford algebras is closed with respect to the tensor product.
\end{abstract}

MSC class: $15 \mathrm{~A} 66$

Key words: Clifford algebra, Quasi Clifford algebras, extended Clifford algebra, tensor product, Cartan-Bott periodicity, complexification.

Considering tensor products of special commutative algebras and general real Clifford algebras, we arrive at extended Clifford algebras $\mathcal{C}(r, s \mid p, q)$. This class of algebras is a subclass of the class of so-called Quasi Clifford algebras [1]. A special case of extended Clifford algebras (in our notations $\mathcal{C}(m, 0 \mid 0, n))$ is used in [2] for coding theory. In particular, the class of extended Clifford algebras contains the class of all Clifford algebras and the classes of complexified Clifford algebras $\mathbb{C} \otimes \mathcal{C}(p, q)$ and hyperbolically complexified Clifford algebras $(\mathbb{R} \oplus \mathbb{R}) \otimes \mathcal{C}(p, q)[6],[7]$. The class of extended Clifford algebras is closed with respect to the operation of tensor product. We develop some results of our previous paper [3] where we study tensor products of Clifford algebras using the Jacobson's approach [4]. A classification of extended Clifford algebras is discussed. We will see that there are five types of extended Clifford algebras and we will find corresponding conditions for integers $r, s, p, q$. 


\section{Clifford algebras $\mathcal{C}(p, q)$, commutative alge- bras $\mathcal{K}(r, s)$, and extended Clifford algebras $\mathcal{C}(r, s \mid p, q)$}

Tensor products of algebras Let $\mathcal{A}$ be a real algebra, which are the real vector space $V$ with a product of elements $A_{1}, A_{2} \rightarrow A_{1} \cdot A_{2}$ that satisfies the distributive condition. And let $\mathcal{B}$ be a real algebra, which are the real vector space $W$ with a product of elements $B_{1}, B_{2} \rightarrow B_{1} * B_{2}$ that satisfies the distributive condition. A tensor product $\mathcal{A} \otimes \mathcal{B}$ of algebras $\mathcal{A}$ and $\mathcal{B}$ is the algebra, which is the tensor product $V \otimes W$ of vector spaces $V$ and $W$ with the operation of multiplication

$$
\left(A_{1} \otimes B_{1}\right) \circ\left(A_{2} \otimes B_{2}\right)=\left(A_{1} \cdot A_{2}\right) \otimes\left(B_{1} * B_{2}\right), \quad \forall A_{1}, A_{2} \in \mathcal{A}, \forall B_{1}, B_{2} \in \mathcal{B} .
$$

Isomorphic real algebras. Real algebras $\mathcal{A}$ and $\mathcal{B}$ of the same dimension are called isomorphic if there exists a one-to-one correspondence (bijection) $T: \mathcal{A} \rightarrow \mathcal{B}$ such that

$$
\begin{aligned}
T\left(\lambda_{1} A_{1}+\lambda_{2} A_{2}\right) & =\lambda_{1} T\left(A_{1}\right)+\lambda_{2} T\left(A_{2}\right), \quad \forall A_{1}, A_{2} \in \mathcal{A}, \quad \forall \lambda_{1}, \lambda_{2} \in \mathbb{R} \\
T\left(A_{1} \cdot A_{2}\right) & =T\left(A_{1}\right) * T\left(A_{2}\right), \quad \forall A_{1}, A_{2} \in \mathcal{A} .
\end{aligned}
$$

Clifford algebras $\mathcal{C}(p, q)$. Consider a real Clifford algebra $\mathcal{C}(p, q)$ of signature $(p, q)$ with $n$ generators $n=p+q, n \geq 1$ (the construction of Clifford algebra is discussed in details in [5]). Let $e$ be the identity element and let $e^{a}, a=1, \ldots, n$ be generators of Clifford algebra $\mathcal{C}(p, q)$,

$$
\begin{gathered}
e^{a} e^{b}+\omega e^{b} e^{a}=0, \quad \forall a, b=1, \ldots, n, \quad a \neq b, \\
\left(e^{a}\right)^{2}=\left\{\begin{aligned}
e, & \text { for } a=1, \ldots, p, \\
-e, & \text { for } a=p+1, \ldots, n,
\end{aligned}\right.
\end{gathered}
$$

where $\omega=1$. The dimension of the Clifford algebra $\mathcal{C}(p, q)$ (as a vector space) is equal to $2^{n}$. Let us define $\mathcal{C}(0,0):=\mathbb{R}$.

Commutative algebras $\mathcal{K}(p, q)$. If we take the above conditions (3) and (44) in the definition of the Clifford algebra $\mathcal{C}(p, q)$ and replace the identity $\omega=1$ by the identity $\omega=-1$, then we arrive at the commutative algebra $\mathcal{K}(p, q)$ 
of signature $(p, q)$ with $n$ generators $n=p+q, n \geq 1$. The dimension of the algebra $\mathcal{K}(p, q)$ (as a vector space) is equal to $2^{n}$. Also define $\mathcal{K}(0,0):=\mathbb{R}$.

Tensor products of Clifford algebras. Let $p_{1}, \ldots, p_{k}, q_{1}, \ldots, q_{k}$ be nonnegative integer numbers and $n_{j}=p_{j}+q_{j}, j=1, \ldots k$ be natural numbers. And let $m$ be the number of odd numbers in the set $n_{1}, \ldots, n_{k}$. Consider the tensor product of Clifford algebras

$$
\mathcal{C} \ell\left(p_{1}, q_{1}\right) \otimes \ldots \otimes \mathcal{C} \ell\left(p_{k}, q_{k}\right)
$$

The following propositions are consequences of Theorem 4 and Theorem 5 from [3]:

1. If $m=0$ or $m=1$, then there exists a nonnegative integer numbers $p, q$ such that $p+q=n=n_{1}+\ldots+n_{k}$ and the tensor product (5) is isomorphic to the Clifford algebra $\mathcal{C}(p, q)$;

2. If $m \geq 2$, then there exists a nonnegative integer numbers $r, s, p, q$ such that $r+s=m, p+q=n=n_{1}+\ldots+n_{k}-m$ and the tensor product (5) is isomorphic to the tensor product

$$
\mathcal{K}(r, s) \otimes \mathcal{C}(p, q)
$$

which can be considered as the Clifford algebra $\mathcal{C}(p, q)$ over the commutative algebra $($ ring $) \mathcal{K}(r, s)$.

Extended Clifford algebras. The above two propositions lead us to consider a class of extended Clifford algebras

$$
\mathcal{C} \ell(r, s \mid p, q):=\mathcal{K}(r, s) \otimes \mathcal{C} \ell(p, q)
$$

where $r, s, p, q$ - nonnegative integer numbers. Any algebra from this class is generated by a set of generators, which is the union of two sets - a set of commuting generators and a set of anticommuting generators. Generators from different sets are commute. As a result we arrive at a class of associative unital algebras dependent on four nonnegative integer numbers. The dimension of this algebra (as a vector space) is equal to $2^{r+s+p+q}$. In particular, the class of extended Clifford algebras contains the class of Clifford algebras $\mathcal{C}(p, q)$, the class of complexified Clifford algebras $\mathbb{C} \otimes \mathcal{C}(p, q)$, and the class of hyperbolically complexified Clifford algebras $(\mathbb{R} \oplus \mathbb{R}) \otimes \mathcal{C}(p, q)$. 
We see that the class of extended Clifford algebras is closed w.r.t. the tensor product, i.e., for any tensor product

$$
\mathcal{C} \ell\left(r_{1}, s_{1} \mid p_{1}, q_{1}\right) \otimes \ldots \otimes \mathcal{C}\left(r_{k}, s_{k} \mid p_{k}, q_{k}\right)
$$

there exist four nonnegative integer numbers $r, s, p, q$ such that $r+s+p+q=$

$\sum_{j=1}^{k}\left(r_{j}+s_{j}+p_{j}+q_{j}\right)$ and the tensor product (7) is isomorphic to the extended Clifford algebra $\mathcal{C}(r, s \mid p, q)$.

\section{Classification of extended Clifford algebras}

Let $M \geq 1, N \geq 0$ be integer numbers. Consider five algebras each of which is a tensor product of Clifford algebras with one or two generators

(I) $\mathcal{C}(1,1)^{N}$;

(II) $\mathcal{C}(0,2) \otimes \mathcal{C}(1,1)^{N-1}, N \geq 1$;

(III) $\quad \mathcal{C}(0,1)^{M} \otimes \mathcal{C}(1,1)^{N}$;

(IV) $\mathcal{C}(1,0)^{M} \otimes \mathcal{C}(1,1)^{N}$;

(V) $\mathcal{C}(1,0)^{M} \otimes \mathcal{C}(0,2) \otimes \mathcal{C}(1,1)^{N-1}, N \geq 1$,

where by $\mathcal{C}(p, q)^{M}$ we denote the tensor product of $M$ pieces of Clifford algebra $\mathcal{C}(p, q)$ and

$$
\mathcal{C}(p, q)^{0}:=\mathcal{C} \ell(0,0) \simeq \mathbb{R} .
$$

Algebras I-V depend on one $(N)$ or two $(M, N)$ nonnegative integer numbers. In the sequel we say about algebras of type I,II,III,IV,V.

Theorem 1 Suppose that $r, s, p, q$ are nonnegative integer numbers and

$$
m=r+s, \quad n=p+q, \quad M=m+\sigma(n), \quad N=\left[\frac{n}{2}\right], \quad t=(p-q) \bmod 8,
$$

where $\left[\frac{n}{2}\right]$ is the integer part of $n / 2$ and

$$
\sigma(n):=n-2\left[\frac{n}{2}\right]= \begin{cases}0, & \text { for even } n \\ 1, & \text { for odd } n\end{cases}
$$


Then the extended Clifford algebra $\mathcal{C}(r, s \mid p, q)$ is isomorphic to one of tensor products of type I-V according to the table

\begin{tabular}{|c||c|c|c|}
\hline type & $r$ & $s$ & $t$ \\
\hline \hline I & 0 & 0 & 0,2 \\
\hline II & 0 & 0 & 4,6 \\
\hline III & $\geq 0$ & $\geq 1$ & $0,1,2,3,4,5,6,7$ \\
& $\geq 0$ & 0 & 3,7 \\
\hline IV & $\geq 0$ & 0 & 1 \\
& $\geq 1$ & 0 & 0,2 \\
\hline V & $\geq 0$ & 0 & 5 \\
& $\geq 1$ & 0 & 4,6 \\
\hline
\end{tabular}

Proof. For Clifford algebras $\mathcal{C}(p, q)$ we have the following Cartan's classification [3]:

$\mathcal{C}(p, q) \simeq \begin{cases}\mathcal{C}(1,1)^{\frac{n}{2}}, & \text { for } p-q=0,2 \bmod 8 ; \\ \mathcal{C}(0,2) \otimes \mathcal{C}(1,1)^{\frac{n-2}{2}}, & \text { for } p-q=4,6 \bmod 8 ; \\ \mathcal{C}(0,1) \otimes \mathcal{C}(1,1)^{\frac{n-1}{2}}, & \text { for } p-q=3,7 \bmod 8 ; \\ \mathcal{C}(1,0) \otimes \mathcal{C}(1,1)^{\frac{n-1}{2}}, & \text { for } p-q=1 \bmod 8 ; \\ \mathcal{C}(1,0) \otimes \mathcal{C}(0,2) \otimes \mathcal{C} \ell(1,1)^{\frac{n-3}{2}}, & \text { for } p-q=5 \bmod 8\end{cases}$

If $r=s=0$ and $t$ is even, then

$$
\mathcal{C} \ell(r, s \mid p, q) \simeq \mathcal{C} \ell(p, q) \simeq \begin{cases}\mathcal{C}(1,1)^{\frac{n}{2}}, & \text { for } t=0,2 \\ \mathcal{C}(0,2) \otimes \mathcal{C}(1,1)^{\frac{n}{2}-1}, & \text { for } t=4,6\end{cases}
$$

That means $\mathcal{C}(0,0 \mid p, q)$ are of the type I for $t=0,2$ and of the type II for $t=4,6$.

If $s=0, r=m \geq 1$, then $\mathcal{K}(r, s) \simeq \mathcal{C} \ell(1,0)^{m}$ and from (9) we get

$$
\mathcal{C} \ell(r, s \mid p, q) \simeq \mathcal{C}(1,0)^{m} \otimes \mathcal{C}(p, q)
$$

\footnotetext{
${ }^{1}$ For types III,IV,V we have two lines in the table that means there are two cases. For example, we have type IV for $r \geq 0, s=0, t=1$ or for $r \geq 1, s=0, t=0,2$.
} 
and

$$
\mathcal{C} \ell(r, s \mid p, q) \simeq \begin{cases}\mathcal{C}(1,0)^{m} \otimes \mathcal{C}(1,1)^{\frac{n}{2}}, & \text { for } t=0,2 ; \\ \mathcal{C}(1,0)^{m} \otimes \mathcal{C}(0,2) \otimes \mathcal{C}(1,1)^{\frac{n-2}{2}}, & \text { for } t=4,6 ; \\ \mathcal{C}(0,1)^{m+1} \otimes \mathcal{C} \ell(1,1)^{\frac{n-1}{2}}, & \text { for } t=3,7 ; \\ \mathcal{C}(1,0)^{m+1} \otimes \mathcal{C} \ell(1,1)^{\frac{n-1}{2}}, & \text { for } t=1 ; \\ \mathcal{C}(1,0)^{m+1} \otimes \mathcal{C}(0,2) \otimes \mathcal{C} \ell(1,1)^{\frac{n-3}{2}}, & \text { for } t=5 .\end{cases}
$$

That means each line at the right hand part of (10) is of the type IV,V,III,IV,V respectively. For $t=3,7$ we use the isomorphism $\mathcal{C}(1,0)^{m} \otimes$ $\mathcal{C}(0,1) \simeq \mathcal{C}(0,1)^{m+1}$.

If $s \geq 1$, then $\mathcal{K}(r, s) \simeq \mathcal{C} \ell(0,1)^{m}$. Using the isomorphism

$$
\mathcal{C} \ell(0,1) \otimes \mathcal{C} \ell(0,2) \simeq \mathcal{C} \ell(0,1) \otimes \mathcal{C}(1,1)
$$

we see that

$$
\mathcal{C} \ell(r, s \mid p, q) \simeq \mathcal{C}(0,1)^{M} \otimes \mathcal{C}(1,1)^{N}
$$

are of the type III for all $t=0,1,2,3,4,5,6,7$. This completes the proof.

Consequence 1. Suppose that we have two sets of four nonnegative integer numbers $r, s, p, q$ and $\dot{r}, \dot{s}, \dot{p}, \dot{q}$ such that $r+s+p+q=\dot{r}+\dot{s}+\not{p}+\dot{q}$. Denote

$$
\begin{aligned}
& m=r+s, \quad n=p+q, \quad M=m+\sigma(n), \quad N=\left[\frac{n}{2}\right], \\
& \dot{m}=\dot{r}+\dot{s}, \quad \dot{n}=\dot{p}+\dot{q}, \quad \dot{M}=\dot{m}+\sigma(\dot{n}), \quad \dot{N}=\left[\frac{\dot{n}}{2}\right] .
\end{aligned}
$$

Extended Clifford algebras $\mathcal{C}(r, s \mid p, q)$ and $\mathcal{C}\left(\dot{r}, s^{\prime} \mid \dot{p}, q\right)$ are isomorphic iff $M^{\prime}=$ $M, N=N$ and both extended Clifford algebras belong to the same type (I$\mathrm{V})$.

Consequence 2. For any fixed natural $n$ all $n+1$ complexified Clifford algebras $\mathbb{C} \otimes \mathcal{C}(p, q), p+q=n$ are isomorphic 2 .

Example. Let us consider the following question3: are there isomorphic algebras among the extended Clifford algebras

$$
\mathcal{C} \ell(3,0 \mid 7,15), \quad \mathcal{C} \ell(4,0 \mid 3,18), \quad \mathcal{C} \ell(5,0 \mid 11,9) ?
$$

\footnotetext{
${ }^{2}$ This proposition is evident for complex Clifford algebras because we can take new generators $e_{k}=i e_{k}$. But for complexified Clifford algebras this proposition is not so evident (at least for me).

${ }^{3}$ The answer to this question can be found using the theory of semisimple algebras you need to do some efforts for this.
} 
We can immediately get the answer on the question. Let $m_{i}, n_{i}, M_{i}, N_{i}, t_{i}$, $i=1,2,3$ be the numbers defined for algebras (11) by formulas (8)

$$
\begin{aligned}
& m_{1}=3, n_{1}=22, M_{1}=3, N_{1}=11, t_{1}=0 \\
& m_{2}=4, n_{2}=21, M_{2}=5, N_{2}=10, t_{2}=1 \\
& m_{3}=5, n_{3}=20, M_{3}=5, N_{3}=10, t_{3}=2 .
\end{aligned}
$$

We see that $M_{2}=M_{3}, \quad N_{2}=N_{3}$ and both extended Clifford algebras $\mathcal{C}(4,0 \mid 3,18), \mathcal{C}(5,0 \mid 11,9)$ belong to the same type IV. According to consequence 1 these algebras are isomorphic.

Acknowledgments. I'm grateful to Prof P.Leopardi for references to important papers [1],[2].

This work was supported by the grant of the Russian Science Foundation RSF 14-50-00005.

\section{References}

[1] Gastineau-Hills H. M., QUASI CLIFFORD ALGEBRAS AND SYSTEMS OF ORTHOGONAL DESIGNS, J. Austral. Math. Soc. (Series A) 32 (1982), 1-23.

[2] G. Susinder Rajan, B. Sundar Rajan, STBCs from Representation of Extended Clifford Algebras, arXiv:0704.2507v1 (2007).

[3] Marchuk N. G., Demonstration Representation and Tensor Products of Clifford Algebras, Proc. Steklov Inst. Math., 290 (2015), 143-154.

[4] Jacobson N, Structure and representations of Jordan algeba, American Math. Soc., Colloquium Publications, vol.39, 1968.

[5] Lounesto P., Clifford Algebras and Spinors, Cambridge Univ. Press (1997, 2001), 338 pages.

[6] Porteous, Ian R., Clifford Algebras and the Classical Groups, Cambridge Studies in Advanced Mathematics, 50, Cambridge University Press, (1995) ISBN 978-0-521-55177-9.

[7] Marchuk N. G., Shirokov D. S., General solutions of one class of field equations, Reports on Mathematical Physics, 78:3 (2016), 305326. 